\title{
Coarse-grained representation of
}

\section{$\beta$-helical protein building blocks}

\author{
David Curcó, ${ }^{1, *}$ Ruth Nussinov ${ }^{2,3}$ and Carlos Alemán ${ }^{4, *}$ \\ ${ }^{1}$ Departament d'Enginyeria Química, Facultat de Química, Universitat de Barcelona, \\ Martí i Franquees 1, Barcelona E-08028, Spain. \\ ${ }^{2}$ Basic Research Program, SAIC-Frederick, Inc. Center for Cancer Research \\ Nanobiology Program, NCI, Frederick, MD 21702, USA \\ ${ }^{3}$ Department of Human Genetics Sackler, Medical School, Tel Aviv University, \\ Tel Aviv 69978, Israel \\ ${ }^{4}$ Departament d'Enginyeria Química, E. T. S. d'Enginyeria Industrial de Barcelona, \\ Universitat Politècnica de Catalunya, Diagonal 647, Barcelona E-08028, Spain.
}
Table S1 Coarse-grained potentials developed to represent the Page S2-S5 interactions between residues $\mathrm{i}, \mathrm{i}+1$

Table S2 Coarse-grained potentials developed to represent the Page S6 intra-residue interactions of the $1 \mathrm{krr}$ building block. 
Table S1. Coarse-grained potentials developed to represent the interactions between residues $i, i+1$ of the $1 \mathrm{krr}$ building block.

ALA-GLY:

$$
\begin{aligned}
& -351.99+78.60 / \mathrm{d}_{\mathrm{clh} 2}^{0.5}+35.58 / \alpha_{\mathrm{olh} 2 \mathrm{o} 2}^{0.5}+5.36 \cdot \varphi_{\mathrm{h} 1 \mathrm{o} 1 \mathrm{~h} 2 \mathrm{o} 2}+ \\
& +24.45 \cdot\left(\mathrm{d}_{\mathrm{h} 2 \mathrm{o} 2}-4.277\right)^{2}+85.06 \cdot\left(\mathrm{d}_{\mathrm{h} 1 \mathrm{c} 1}-2.74\right)^{2}+85.26 \cdot \mathrm{d}_{\mathrm{olh} 2}+ \\
& +29.22 \cdot\left(\alpha_{\mathrm{o} 1 \mathrm{~h} 2 \mathrm{o} 2}-0.817\right)^{2}+6.22 / \mathrm{d}_{\mathrm{c} 1 \mathrm{o} 1}^{0.5}
\end{aligned}
$$

ASN-ASN

$$
\begin{aligned}
& 4.48-1.72 \cdot\left(\alpha_{\mathrm{x} 1 \mathrm{c} 1 \mathrm{~h} 1}-1.322\right)^{2}+1.35 \cdot\left(\mathrm{d}_{\mathrm{c} 2 \mathrm{~h} 1}-5.634\right)^{2}-38.81 / \mathrm{d}_{\mathrm{x} 1 \mathrm{~h} 2}^{2}- \\
& -77.09 \cdot\left(\alpha_{\mathrm{o} 1 \mathrm{~h} 2 \mathrm{o} 2}-0.868\right)^{2}+5.01 \cdot\left(\alpha_{\mathrm{c} 1 \mathrm{~h} 1 \mathrm{o} 1}-0.912\right)^{2}+ \\
& +1.06 \cdot\left(\alpha_{\mathrm{h} 2 \mathrm{c} 2 \mathrm{x} 2}-1.227\right)^{2}+34.67 / \mathrm{d}_{\mathrm{x} 1 \mathrm{x} 2}^{2}-0.02 \cdot\left(\varphi_{\mathrm{x} 1 \mathrm{c} 1 \mathrm{c} 2 \mathrm{x} 2}-6.283\right)^{2}- \\
& -53.75 \cdot\left(\alpha_{\mathrm{h} 1 \mathrm{o} 1 \mathrm{~h} 2}-1.635\right)^{2}
\end{aligned}
$$

ASN-PRO

$$
\begin{aligned}
& -611.2+0.076 \cdot\left(\varphi_{\mathrm{x} 1 \mathrm{c} 1 \mathrm{olh} 1}-6.281\right)^{2}+0.49 \cdot \varphi_{\mathrm{h} 1 \mathrm{olh} 2 \mathrm{c} 2}+ \\
& +1.91 \cdot\left(\varphi_{\mathrm{c} 1 \mathrm{~h} 1 \mathrm{olh} 2}-5.805\right)^{2}+165.52 \cdot \mathrm{d}_{\mathrm{o} 1 \mathrm{~h} 2}+1.11 \cdot\left(\mathrm{d}_{\mathrm{clh} 2}-4.977\right)^{2}+ \\
& 3.30 \cdot \mathrm{d}_{\mathrm{h} 1 \mathrm{o} 2}+7.41 \cdot \alpha_{\mathrm{h} 1 \mathrm{olh} 2}+0.69 \cdot\left(\mathrm{d}_{\mathrm{c} 1 \mathrm{~h} 2}-3.197\right)^{2}
\end{aligned}
$$

ASN-SER

$$
\begin{aligned}
& -164.4+43.8 \cdot \mathrm{d}_{\mathrm{o} 1 \mathrm{~h} 2}+33.5 / \mathrm{d}_{\mathrm{clx} 1}^{0.5}+9.5 \cdot\left(\alpha_{\mathrm{o} 1 \mathrm{~h} 2 \mathrm{o} 2}-1.42\right)^{2}+ \\
& +131.2 \cdot\left(\mathrm{d}_{\mathrm{c} 2 \mathrm{x} 2}-1.093\right)^{2}+3.5 \cdot\left(\mathrm{d}_{\mathrm{c} 2 \times 2}-1.258\right)^{2}+0.71 / \varphi_{\mathrm{x} 1 \mathrm{c} 1 \mathrm{olh} 1}^{0.5}+ \\
& +0.41 \cdot \mathrm{d}_{\mathrm{x} 2 \mathrm{~h} 1}+4.0 / \alpha_{\mathrm{x} 1 \mathrm{c} 1 \mathrm{~h} 1}^{2}
\end{aligned}
$$

ASN-VAL

$$
\begin{aligned}
& -306.3+-47.61 /\left(\mathrm{d}_{\mathrm{clc} 2}\right)^{0.5}+104.69 \cdot \mathrm{d}_{\mathrm{olh} 2}+3.9 \cdot\left(\alpha_{\mathrm{o} 1 \mathrm{~h} 2 \mathrm{o} 2}-1.57\right)^{2}- \\
& -0.06 \cdot\left(\varphi_{\mathrm{x} 1 \mathrm{c} 1 \mathrm{olh} 1}-0.287\right)^{2}+12.9 \cdot\left(\varphi_{\mathrm{h} 1 \mathrm{olh} 2 \mathrm{o} 2}-1.997\right)^{2}+ \\
& +48.5 \cdot\left(\alpha_{\mathrm{h} 1 \mathrm{olh} 2}-0.921\right)^{2}+0.34 \cdot\left(\mathrm{d}_{\mathrm{clo} 2}-7.125\right)^{2}- \\
& -0.007 \cdot\left(\varphi_{\mathrm{x} 1 \mathrm{c} 1 \mathrm{c} 2 \mathrm{x} 2}-6.281\right)^{2}
\end{aligned}
$$

ASP-ASN

$$
\begin{aligned}
& -530.2+1.71 *\left(\mathrm{~d}_{\mathrm{c} 2 \mathrm{o} 2}-4.421\right)^{2}+166.7 \cdot \mathrm{d}_{\mathrm{o} 1 \mathrm{~h} 2}+ \\
& +2.3 \cdot\left(\varphi_{\mathrm{c} 1 \mathrm{~h} 1 \mathrm{olh} 2}-5.052\right)^{2}++10.2 / \alpha_{\mathrm{o} 1 \mathrm{~h} 2 \mathrm{o} 2}^{0.5}+0.44 \cdot\left(\mathrm{d}_{\mathrm{x} 2 \mathrm{~h} 1}-8.649\right)^{2}+ \\
& +7.6 / \alpha_{\mathrm{h} 2 \mathrm{c} 2 \times 2}^{2}+0.94 \cdot\left(\mathrm{d}_{\mathrm{h} 1 \mathrm{c} 1}-3.478\right)^{2}+2.81 \cdot \mathrm{d}_{\mathrm{c} 2 \mathrm{o} 1}
\end{aligned}
$$

GLY-ALA

$$
\begin{aligned}
& -399.5+6.40 / \alpha_{\mathrm{olh} 202}^{2}+2.31 \cdot \varphi_{\mathrm{h} 1 \mathrm{olh} 2 \mathrm{o} 2}+123.7 \cdot \mathrm{d}_{\mathrm{o} 1 \mathrm{~h} 2}+ \\
& +22.1 \cdot\left(\alpha_{\mathrm{h} 1 \mathrm{olh} 2}-1.645\right)^{2}+6.31 \cdot\left(\mathrm{d}_{\mathrm{c} 2 \mathrm{o} 1}-4.705\right)^{2}-0.15 \cdot\left(\mathrm{d}_{\mathrm{c} 2 \mathrm{o} 2}-3.31\right)^{2}
\end{aligned}
$$


GLY-ASN

$$
\begin{aligned}
& -393.3+3.86 \cdot \mathrm{d}_{\mathrm{h} 2 \mathrm{c} 2}-18.4 \cdot \alpha_{\mathrm{o} 1 \mathrm{~h} 2 \mathrm{o} 2}+127.6 \cdot \mathrm{d}_{\mathrm{o} 1 \mathrm{~h} 2}+0.63 / \varphi_{\mathrm{o} 1 \mathrm{~h} 2 \mathrm{c} 2 \mathrm{x} 2}^{2}- \\
& -31.0 \cdot\left(\mathrm{d}_{\mathrm{h} 2 \mathrm{o} 2}-3.221\right)^{2}+12.4 / \alpha_{\mathrm{h} 2 \mathrm{c} 2 \mathrm{x} 2}^{2}-1.37 \cdot\left(\alpha_{\mathrm{c} 2 \mathrm{~h} 2 \mathrm{o} 2}-0.912\right)^{2}+ \\
& +3.9 \cdot\left(\mathrm{d}_{\mathrm{h} 1 \mathrm{o} 1}-3.111\right)^{2}
\end{aligned}
$$

GLY-ASP

$1.77+47.87 \cdot\left(\alpha_{\mathrm{o} 1 \mathrm{~h} 2 \mathrm{o} 2}-1.272\right)^{2}+1.15 \cdot\left(\mathrm{d}_{\mathrm{h} 1 \mathrm{o} 2}-4.442\right)^{2}-$

$-2.77 \cdot\left(\alpha_{\mathrm{olh} 2 \mathrm{c} 2}-1.609\right)^{2}+39.11 \cdot\left(\alpha_{\mathrm{h} 1 \mathrm{olh} 2}-1.535\right)^{2}-$

$-6.02 \cdot\left(\varphi_{\mathrm{h} 1 \mathrm{o} 1 \mathrm{~h} 2 \mathrm{o} 2}-1.524\right)^{2}-1.09 \cdot\left(\mathrm{d}_{\mathrm{h} 1 \mathrm{o} 2}-5.996\right)^{2}+$

$23.15 \cdot\left(\mathrm{d}_{\mathrm{h} 2 \mathrm{o} 2}-3.17\right)^{2}+9.95 \cdot\left(\mathrm{d}_{\mathrm{h} 2 \mathrm{o} 2}-3.517\right)^{2}$

GLY-SER

$$
\begin{aligned}
& -3.88+0.076 \cdot \mathrm{d}_{\mathrm{c} 2 \mathrm{~h} 1}+0.74 \cdot\left(\alpha_{\mathrm{o} 2 \mathrm{c} 2 \mathrm{x} 2}-0.843\right)^{2}+6.31 / \alpha_{\mathrm{o} 1 \mathrm{~h} 2 \mathrm{o} 2}^{2}+ \\
& +0.0024 * \varphi_{\mathrm{o} 1 \mathrm{~h} 2 \mathrm{c} 2 \times 2}^{2}-1.978 / \alpha_{\mathrm{h} 2 \mathrm{c} 2 \times 2}^{2}+1.35 \cdot\left(\mathrm{d}_{\mathrm{c} 2 \mathrm{o} 2}-4.001\right)^{2}+ \\
& +0.23 \cdot\left(\mathrm{d}_{\mathrm{h} 1 \mathrm{o} 2}-4.818\right)^{2}+54.0 \cdot\left(\mathrm{d}_{\mathrm{c} 2 \times 2}-1.182\right)^{2}
\end{aligned}
$$

GLY-VAL

$$
\begin{aligned}
& -311.11+33.55 \cdot\left(\alpha_{\mathrm{o} 1 \mathrm{~h} 2 \mathrm{o} 2}-1.246\right)^{2}+4.01 / \alpha_{\mathrm{h} 1 \mathrm{o} 1 \mathrm{~h} 2}+ \\
& +9.46 \cdot\left(\mathrm{d}_{\mathrm{h} 2 \mathrm{o} 2}-3.467\right)^{2}+95.6 \cdot \mathrm{d}_{\mathrm{o} 1 \mathrm{~h} 2}+0.63 \cdot \mathrm{d}_{\mathrm{h} 2 \mathrm{c} 2}+9.76 \cdot\left(\mathrm{d}_{\mathrm{h} 1 \mathrm{o} 1}-3.993\right)^{2}
\end{aligned}
$$

HSD-VAL

$$
\begin{aligned}
& -2.68-4.56 \cdot\left(\alpha_{\mathrm{c} 2 \mathrm{~h} 202}-1.074\right)^{2}-4.22 \cdot\left(\varphi_{\mathrm{h} 1 \mathrm{o} 1 \mathrm{~h} 2 \mathrm{o} 2}-2.914\right)^{2}- \\
& -2.70 \cdot\left(\mathrm{d}_{\mathrm{h} 2 \mathrm{o} 2}-3.035\right)^{2}+5.76 / \alpha_{\mathrm{h} 1 \mathrm{olh} 2}^{0.5}-5.28 / \varphi_{\mathrm{clh} 1 \mathrm{olh} 2}^{2}- \\
& -14.68 \cdot\left(\mathrm{d}_{\mathrm{h} 1 \mathrm{o} 1}-4.488\right)^{2}--3.21 \cdot\left(\alpha_{\mathrm{o} 1 \mathrm{~h} 2 \mathrm{o} 2}-1.485\right)^{2}+ \\
& +1.25 \cdot\left(\alpha_{\mathrm{o} 1 \mathrm{~h} 2 \mathrm{oc} 2}-1.796\right)^{2}
\end{aligned}
$$

ILE-ASN

$$
\begin{aligned}
& -96.2+0.5 \cdot \mathrm{d}_{\mathrm{c} 2 \mathrm{o} 2}+1.91 \cdot\left(\mathrm{d}_{\mathrm{h} 2 \mathrm{c} 2}-2.18\right)^{2}-10.96 / \alpha_{\mathrm{h} 2 \mathrm{c} 2 \times 2}^{2}+ \\
& +53.13 / \mathrm{d}_{\mathrm{c} 2 \times 2}^{0.5}+9.71 \cdot\left(\mathrm{d}_{\mathrm{h} 2 \mathrm{o} 2}-3.191\right)^{2}+19.12 / \varphi_{\mathrm{h} 1 \mathrm{o} 1 \mathrm{~h} 2 \mathrm{o} 2}^{0.5}+ \\
& +0.35 \cdot \mathrm{d}_{\mathrm{clh} 2}+0.25 \cdot\left(\mathrm{d}_{\mathrm{h} 1 \mathrm{o} 2}-6.551\right)^{2}+0.58 \cdot \varphi_{\mathrm{c} 1 \mathrm{olh} 2 \mathrm{o} 2}+ \\
& +6.7 / \alpha_{\mathrm{o} 1 \mathrm{~h} 2 \mathrm{c} 2}^{0.5}+42.7 / \alpha_{\mathrm{h} 2 \mathrm{c} 2 \mathrm{x} 2}^{0.5}
\end{aligned}
$$

ILE-GLY

$$
\begin{aligned}
& 2.09+5.42 \cdot\left(\mathrm{d}_{\mathrm{h} 1 \mathrm{o} 1}-2.445\right)^{2}-7.91 \cdot\left(\alpha_{\mathrm{c} 1 \mathrm{~h} 1 \mathrm{o} 1}-1.698\right)^{2}+8.27 / \alpha_{\mathrm{o} 1 \mathrm{~h} 2 \mathrm{o} 2}^{2}+ \\
& +0.42 \cdot\left(\mathrm{d}_{\mathrm{c} 1 \mathrm{o} 2}-6.279\right)^{2}+14.36 \cdot\left(\mathrm{d}_{\mathrm{h} 2 \mathrm{o} 2}-3.589\right)^{2}-4.06 \cdot\left(\mathrm{d}_{\mathrm{c} 1 \mathrm{x} 1}-0.519\right)^{2}- \\
& -3.23 \cdot\left(\alpha_{\mathrm{h} 1 \mathrm{olh} 2}-1.742\right)^{2}-0.33 \cdot\left(\mathrm{d}_{\mathrm{x} 1 \mathrm{o} 2}-3.971\right)^{2}
\end{aligned}
$$


ILE-THR

$$
\begin{aligned}
& 5.87-50.51 / \mathrm{d}_{\mathrm{x} 2 \mathrm{o} 1}^{2}-19.05 \cdot\left(\alpha_{\mathrm{o} 1 \mathrm{~h} 2 \mathrm{o} 2}-2.12\right)^{2}+3.52 \cdot\left(\varphi_{\mathrm{h} 1 \mathrm{olh} 2 \mathrm{c} 2}-4.42\right)^{2}+ \\
& +0.80 \cdot\left(\alpha_{\mathrm{o} 2 \mathrm{c} 2 \mathrm{x} 2}-0.66\right)^{2}+1.52 / \alpha_{\mathrm{h} 2 \mathrm{c} 2 \mathrm{x} 2}^{2}+0.07 \cdot \varphi_{\mathrm{o} 1 \mathrm{~h} 2 \mathrm{c} 2 \mathrm{x} 2}^{2}+13.19 / \mathrm{d}_{\mathrm{c} 1 \mathrm{o} 1}- \\
& -0.098 \cdot \varphi_{\mathrm{x} 1 \mathrm{c} 1 \mathrm{c} 2 \times 2}-6.01 \cdot\left(\mathrm{d}_{\mathrm{c} 2 \mathrm{o} 2}-3.118\right)^{2}-5.66 \cdot\left(\mathrm{d}_{\mathrm{c} 2 \mathrm{o} 2}-4.155\right)^{2}- \\
& -0.0065 \cdot \varphi_{\mathrm{x} 1 \mathrm{c} 1 \mathrm{olh} 2}^{2}
\end{aligned}
$$

ILE-VAL

$$
\begin{aligned}
& -24.67+4.36 \cdot\left(\mathrm{d}_{\mathrm{h} 2 \mathrm{c} 2}-3.073\right)^{2}+33.23 / \alpha_{\mathrm{o} 1 \mathrm{~h} 2 \mathrm{o} 2}^{2}+10.07 \cdot \alpha_{\mathrm{h} 1 \mathrm{olh} 2}+ \\
& +0.195 \cdot\left(\mathrm{d}_{\mathrm{x} 1 \mathrm{o} 2}-5.352\right)^{2}+1.4 \cdot\left(\varphi_{\mathrm{c} 1 \mathrm{olh} 2 \mathrm{o} 2}-1.763\right)^{2}-4.12 \cdot\left(\mathrm{d}_{\mathrm{h} 1 \mathrm{o} 1}-2.856\right)^{2}+ \\
& +1.21 \cdot\left(\mathrm{d}_{\mathrm{c} 2 \mathrm{o} 2}-2.68\right)^{2}+5.11 \cdot\left(\alpha_{\mathrm{c} 1 \mathrm{~h} 1 \mathrm{o} 1}-1.609\right)^{2}
\end{aligned}
$$

PRO-GLY

$$
\begin{aligned}
& -404.7+1.07 \cdot \varphi_{\mathrm{c} 1 \mathrm{~h} 1 \mathrm{olh} 2}+15.63 \cdot \mathrm{d}_{\mathrm{h} 2 \mathrm{o} 2}+1.02 \cdot \mathrm{d}_{\mathrm{h} 1 \mathrm{o} 1}+38.30 \cdot \alpha_{\mathrm{olh} 2 \mathrm{o} 2}+ \\
& +88.43 \cdot \mathrm{d}_{\mathrm{olh} 2}+1.14 \cdot \varphi_{\mathrm{h} 1 \mathrm{olh} 2 \mathrm{o} 2}+99.21 \cdot\left(\mathrm{d}_{\mathrm{clh} 2}-1.765\right)^{2}
\end{aligned}
$$

PRO-ILE

$$
\begin{aligned}
& -385.97+7.21 \cdot \varphi_{\mathrm{c} 1 \mathrm{o} 1 \mathrm{~h} 2 \mathrm{o} 2}+5.10 \cdot\left(\alpha_{\mathrm{o} 1 \mathrm{~h} 2 \mathrm{o} 2}-1.735\right)^{2}+2.15 \cdot \varphi_{\mathrm{c} 1 \mathrm{~h} 1 \mathrm{olh} 2}+ \\
& +0.57 \cdot \mathrm{d}_{\mathrm{c} 2 \mathrm{~h} 1}+111.46 \cdot \mathrm{d}_{\mathrm{o} 1 \mathrm{~h} 2}+1.0 / \alpha_{\mathrm{olh} 2 \mathrm{c} 2}^{2}+1.05 \cdot \alpha_{\mathrm{x} 1 \mathrm{c} 1 \mathrm{~h} 1}+1.57 \cdot \mathrm{d}_{\mathrm{h} 2 \mathrm{o} 2}
\end{aligned}
$$

\section{SER-HSD}

$$
\begin{aligned}
& -0.335-39.64 / \mathrm{d}_{\mathrm{x} 1 \times 2}^{2}+3.85 \cdot\left(\mathrm{d}_{\mathrm{c} 2 \mathrm{o} 2}-4.765\right)^{2}-47.45 \cdot\left(\alpha_{\mathrm{o} 1 \mathrm{~h} 2 \mathrm{o} 2}-0.834\right)^{2}- \\
& -30.42 \cdot\left(\mathrm{d}_{\mathrm{h} 2 \mathrm{o} 2}-4.488\right)^{2}-9.57 \cdot\left(\varphi_{\mathrm{c} 1 \mathrm{olh} 2 \mathrm{o} 2}-2.971\right)^{2}+ \\
& +6.93 \cdot\left(\alpha_{\mathrm{o} 1 \mathrm{~h} 2 \mathrm{c} 2}-0.768\right)^{2}-0.13 \cdot\left(\varphi_{\mathrm{x} 1 \mathrm{c} 1 \mathrm{olh} 1}-1.314\right)^{2}-0.43 \cdot\left(\mathrm{d}_{\mathrm{c} 1 \mathrm{c} 2}-3.464\right)^{2}
\end{aligned}
$$

\section{SER-ILE}

$$
\begin{aligned}
& -449.56+2.61 / \alpha_{\mathrm{x} 1 \mathrm{c} 1 \mathrm{o} 1}^{2}+2.57 \cdot \mathrm{d}_{\mathrm{c} 1 \mathrm{c} 2}+0.56 \cdot \varphi_{\mathrm{x} 1 \mathrm{c} 1 \mathrm{o} 1 \mathrm{~h} 1}+131.59 \cdot \mathrm{d}_{\mathrm{o} 1 \mathrm{~h} 2}+ \\
& +0.0054 \cdot \varphi_{\mathrm{o} 1 \mathrm{~h} 2 \mathrm{c} 2 \mathrm{x} 2}^{2}+5.60 \cdot \alpha_{\mathrm{c} 1 \mathrm{~h} 1 \mathrm{o} 1}+0.97 \cdot \mathrm{d}_{\mathrm{h} 1 \mathrm{o} 2}+4.58 \cdot\left(\mathrm{d}_{\mathrm{h} 2 \mathrm{o} 2}-2.467\right)^{2}+ \\
& +0.315 / \varphi_{\mathrm{x} 1 \mathrm{c} 1 \mathrm{o} 1 \mathrm{~h} 1}++0.67 \cdot\left(\mathrm{d}_{\mathrm{h} 2 \mathrm{c} 2}-4.137\right)^{2}+9.52 / \mathrm{d}_{\mathrm{c} 2 \mathrm{o} 2}^{0.5}+ \\
& +64.22 \cdot\left(\mathrm{d}_{\mathrm{c} 1 \mathrm{x} 1}-1.082\right)^{2}
\end{aligned}
$$

SER-VAL

$$
\begin{aligned}
& -8.74-16.11 \cdot\left(\alpha_{\mathrm{c} 2 \mathrm{~h} 2 \mathrm{o} 2}-1.127\right)^{2}+3.03 \cdot \mathrm{d}_{\mathrm{c} 202}-0.34 / \alpha_{\mathrm{x} 1 \mathrm{c} 1 \mathrm{~h} 1}+ \\
& +1.66 \cdot\left(\varphi_{\mathrm{h} 1 \mathrm{o} 1 \mathrm{~h} 2 \mathrm{c} 2}-3.473\right)^{2}+1.51 \cdot \mathrm{d}_{\mathrm{c} 2 \mathrm{o} 1}-7.01 \cdot\left(\alpha_{\mathrm{c} 1 \mathrm{o} 1 \mathrm{~h} 2}-0.965\right)^{2}- \\
& -5.04 \cdot \mathrm{d}_{\mathrm{c} 1 \mathrm{x} 1}+0.58 \cdot\left(\alpha_{\mathrm{x} 1 \mathrm{c} 1 \mathrm{o} 1}-2.649\right)^{2}-0.80 \cdot \mathrm{d}_{\mathrm{h} 1 \mathrm{o} 1}+ \\
& +3.22 \cdot\left(\mathrm{d}_{\mathrm{h} 2 \mathrm{o} 2}-2.817\right)^{2}-15.38 / \mathrm{d}_{\mathrm{x} 102}^{2}-3.91 \cdot\left(\varphi_{\mathrm{h} 1 \mathrm{o} 1 \mathrm{~h} 2 \mathrm{c} 2}-4.069\right)^{2}
\end{aligned}
$$


THR-ILE

$$
\begin{aligned}
& -48.69+4.78 \cdot \mathrm{d}_{\mathrm{h} 2 \mathrm{o} 2}+2.83 \cdot \mathrm{d}_{\mathrm{c} 1 \times 2}+129.22 / \mathrm{d}_{\mathrm{c} 2 \mathrm{~h} 1}+ \\
& +0.53 \cdot\left(\mathrm{d}_{\mathrm{x} 1 \mathrm{o} 2}-7.088\right)^{2}-4.01 \cdot \varphi_{\mathrm{h} 1 \mathrm{o} 1 \mathrm{~h} 2 \mathrm{c} 2}+1.79 \cdot\left(\mathrm{d}_{\mathrm{c} 2 \mathrm{o} 2}-4.601\right)^{2}+ \\
& +1.14 \cdot \mathrm{d}_{\mathrm{c} 1 \mathrm{o} 1}-2.02 \cdot\left(\varphi_{\mathrm{c} 1 \mathrm{olh} 2 \mathrm{o} 2}-1.638\right)^{2}+7.03 \cdot\left(\mathrm{d}_{\mathrm{c} 2 \mathrm{x} 2}-0.08\right)^{2}
\end{aligned}
$$

TRP-ILE

$$
\begin{aligned}
& -26.35+0.64 \cdot \mathrm{d}_{\mathrm{x} 1 \times 2}+48.68 / \varphi_{\mathrm{h} 1 \mathrm{o} 1 \mathrm{~h} 2 \mathrm{c} 2}^{0.5}+0.135 \cdot\left(\alpha_{\mathrm{h} 2 \mathrm{c} 2 \times 2}-0.467\right)^{2}+ \\
& +176.85 \cdot\left(\mathrm{d}_{\mathrm{c} 1 \times 1}-1.134\right)^{2}+0.039 \cdot \varphi_{\mathrm{o} 1 \mathrm{~h} 2 \mathrm{c} 2 \mathrm{x} 2}+1.11 \cdot\left(\mathrm{d}_{\mathrm{h} 2 \mathrm{c} 2}-2.585\right)^{2}+ \\
& +2.87 \cdot\left(\mathrm{d}_{\mathrm{h} 2 \mathrm{o} 2}-2.639\right)^{2}+4.44 \cdot\left(\mathrm{d}_{\mathrm{h} 1 \mathrm{o} 1}-2.907\right)^{2}+6.95 / \mathrm{d}_{\mathrm{c} 1 \mathrm{o} 1}^{2}
\end{aligned}
$$

VAL-ILE

$$
\begin{aligned}
& -45.36+18.21 \cdot \mathrm{d}_{\mathrm{o} 1 \mathrm{~h} 2}+0.0373 \cdot \mathrm{d}_{\mathrm{x} 2 \mathrm{~h} 1}+0.615 \cdot\left(\mathrm{d}_{\mathrm{h} 2 \mathrm{c} 2}-2.685\right)^{2}- \\
& -3.78 \cdot\left(\mathrm{d}_{\mathrm{h} 2 \mathrm{o} 2}-3.001\right)^{2}-53.33 / \varphi_{\mathrm{c} 1 \mathrm{~h} 1 \mathrm{olh} 2}+0.051 \cdot\left(\alpha_{\mathrm{h} 2 \mathrm{c} 2 \mathrm{x} 2}-0.317\right)^{2}+ \\
& +1.97 \cdot\left(\mathrm{d}_{\mathrm{c} 1 \mathrm{o} 1}-4.201\right)^{2}+0.094 / \alpha_{\mathrm{h} 2 \mathrm{c} 2 \times 2}^{2}
\end{aligned}
$$

VAL-THR

$$
\begin{aligned}
& 0.60+2.74 \cdot\left(\varphi_{\mathrm{c} 1 \mathrm{~h} 1 \mathrm{ol} 2 \mathrm{~h}}-4.448\right)^{2}-1.72 \cdot\left(\mathrm{d}_{\mathrm{c} 2 \mathrm{o} 2}-2.811\right)^{2}+ \\
& +19.41 / \mathrm{d}_{\mathrm{h} 2 \mathrm{c} 2}^{2}+2.65 / \alpha_{\mathrm{h} 2 \mathrm{c} 2 \times 2}^{2}+0.76 \cdot\left(\alpha_{\mathrm{h} 2 \mathrm{c} 2 \times 2}-0.672\right)^{2}- \\
& -1.09 / \alpha_{\mathrm{o} 2 \mathrm{c} 2 \times 2}^{2}-0.72 \cdot\left(\mathrm{d}_{\mathrm{c} 2 \mathrm{o} 1}-5.129\right)^{2}
\end{aligned}
$$

VAL-TRP

$$
\begin{aligned}
& 7.06-0.30 \cdot\left(\mathrm{d}_{\mathrm{h} 2 \mathrm{c} 2}-5.817\right)^{2}+10.24 \cdot\left(\varphi_{\mathrm{c} 1 \mathrm{~h} 1 \mathrm{olh} 2}-5.236\right)^{2}- \\
& -38.92 \cdot\left(\alpha_{\mathrm{o} 1 \mathrm{~h} 2 \mathrm{o} 2}-1.686\right)^{2}-0.69 \cdot\left(\mathrm{d}_{\mathrm{h} 1 \mathrm{c} 1}-3.175\right)^{2}+ \\
& +5.33 \cdot\left(\varphi_{\mathrm{h} 1 \mathrm{o} 1 \mathrm{~h} 2 \mathrm{o} 2}-2.165\right)^{2}-0.54 \cdot\left(\alpha_{\mathrm{o} 2 \mathrm{c} 2 \times 2}-1.679\right)^{2}-3.16 \cdot\left(\mathrm{d}_{\mathrm{h} 1 \mathrm{o} 2}-7.269\right)^{2}
\end{aligned}
$$

VAL-VAL

$$
\begin{aligned}
& -31.82+10.95 \cdot\left(\varphi_{\mathrm{c} 1 \mathrm{~h} 1 \mathrm{olh} 2}-5.013\right)^{2}+5.23 \cdot\left(\mathrm{d}_{\mathrm{h} 2 \mathrm{c} 2}-2.943\right)^{2}+ \\
& +3.28 \cdot \varphi_{\mathrm{h} 1 \mathrm{olh} 2 \mathrm{c} 2}++4.06 \cdot \mathrm{d}_{\mathrm{h} 2 \mathrm{o} 2}+2.50 \cdot \mathrm{d}_{\mathrm{h} 1 \mathrm{o} 1}+0.42 \cdot\left(\mathrm{d}_{\mathrm{c} 1 \mathrm{o} 1}-3.093\right)^{2}+ \\
& +1.80 \cdot\left(\mathrm{d}_{\mathrm{h} 1 \mathrm{c} 1}-2.574\right)^{2}+10.07 \cdot\left(\alpha_{\mathrm{c} 1 \mathrm{olh} 2}-0.888\right)^{2}
\end{aligned}
$$


Table S2. Coarse-grained potentials developed to represent the intra-residue interactions of the $1 \mathrm{krr}$ building block.

\begin{tabular}{|c|c|}
\hline & CG potential \\
\hline ALA & $-45.27+8.16 / \alpha_{\mathrm{cho}}^{2}+473.46 / \mathrm{d}_{\mathrm{ch}}^{2}-0.5 \cdot \mathrm{d}_{\mathrm{ho}}^{2}+9.34 \cdot \alpha_{\mathrm{cho}}^{2}$ \\
\hline ASN & $\begin{array}{l}-37.93-0.76 \cdot \mathrm{d}_{\mathrm{ho}}^{2}-0.63 \cdot \cos ^{2}\left(\varphi_{\text {xcho }}\right)-30.13 / \mathrm{d}_{\mathrm{ch}}^{2}+12.57 \cdot \mathrm{d}_{\mathrm{cx}}^{2}- \\
-8.15 / \mathrm{d}_{\mathrm{ox}}^{0.5}-2.73 \cdot \alpha_{\mathrm{cho}}^{2}-1.60 \cdot \cos \left(\varphi_{\mathrm{xcho}}\right)+0.91 \cdot \alpha_{\mathrm{xch}}^{2}+8.40 / \alpha_{\mathrm{xch}}^{2}- \\
-0.26 \cdot \mathrm{d}_{\mathrm{ch}}^{2}-0.45 \cdot \cos ^{2}\left(\varphi_{\mathrm{xcoh}}\right)+0.59 \cdot \cos \left(\varphi_{\mathrm{xcoh}}\right)\end{array}$ \\
\hline ASP & $\begin{array}{l}82.81-22.02 \cdot \cos \left(\varphi_{\mathrm{xcoh}}\right)+19.02 / \alpha_{\mathrm{xch}}^{2}+139.05 / \mathrm{d}_{\mathrm{ho}}^{2}- \\
-0.76 \cdot \cos ^{2}\left(\varphi_{\mathrm{xcoh}}\right)-495.99 / \mathrm{d}_{\mathrm{oc}}^{2}-4.01 \cdot \alpha_{\mathrm{xch}}^{2}-82.45 \cdot \mathrm{d}_{\mathrm{cx}}+ \\
+6.60 \cdot \cos ^{2}\left(\varphi_{\mathrm{xcho}}\right)-8.87 \cdot \cos \left(\varphi_{\mathrm{xcho}}\right)+38.47 / \alpha_{\mathrm{cho}}^{2}\end{array}$ \\
\hline GLY & $383.04-88.56 \cdot \mathrm{d}_{\mathrm{ho}}+6.83 \cdot \mathrm{d}_{\mathrm{ho}}^{2}-445.65 / \mathrm{d}_{\mathrm{ho}}$ \\
\hline HSD & $\begin{array}{l}73.06-2.24 \cdot \cos ^{2}\left(\varphi_{\mathrm{xcoh}}\right)-3.85 \cdot \alpha_{\mathrm{xch}}^{2}-14.50 \cdot \alpha_{\mathrm{cho}}^{2}+ \\
+1150.34 / \mathrm{d}_{\mathrm{ho}}^{2}+230.99 / \mathrm{d}_{\mathrm{cx}}^{2}+0.41 / \cos ^{2}\left(\varphi_{\mathrm{xcho}}\right)-561.03 / \mathrm{d}_{\mathrm{ox}}^{2}+ \\
+418.52 / \mathrm{d}_{\mathrm{hx}}^{2}+1.58 \cdot \mathrm{d}_{\mathrm{ch}}^{2}-1.72 \cdot \alpha_{\mathrm{xco}}^{2}\end{array}$ \\
\hline ILE & $\begin{array}{l}29.65-0.76 \cdot \mathrm{d}_{\mathrm{hx}}^{2}+0.33 \cdot \mathrm{d}_{\mathrm{ho}}^{2}+1.02 \cdot \cos \left(\varphi_{\mathrm{xcoh}}\right)+0.76 \cdot \mathrm{d}_{\mathrm{oc}}^{2}- \\
-2.69 / \alpha_{\mathrm{xch}}^{0.5}+0.84 \cdot \cos ^{2}\left(\varphi_{\mathrm{xcho}}\right)+0.19 \cdot \cos \left(\varphi_{\mathrm{xcho}}\right)+ \\
+0.004 / \alpha_{\mathrm{xch}}^{2}+0.48 \cdot \cos ^{2}\left(\varphi_{\mathrm{xcoh}}\right)-0.71 / \alpha_{\mathrm{xco}}^{0.5}+0.00072 / \alpha_{\mathrm{xco}}^{2}+ \\
+13.73 / \alpha_{\mathrm{cho}}^{2}\end{array}$ \\
\hline PRO & $\begin{array}{l}34.19-6.18 \cdot \mathrm{d}_{\mathrm{cx}}+108.67 / \mathrm{d}_{\mathrm{ox}}^{2}+14.43 \cdot \cos ^{2}\left(\varphi_{\mathrm{xcoh}}\right)+ \\
+49.39 / \mathrm{d}_{\mathrm{ch}}^{2}+0.40 / \mathrm{d}_{\mathrm{cx}}^{2}-17.55 / \alpha_{\mathrm{xco}}^{2}+12.76 \cdot \cos \left(\varphi_{\mathrm{xcoh}}\right)- \\
-19.11 / \alpha_{\mathrm{xch}}^{2}-118.75 / \mathrm{d}_{\mathrm{ho}}^{2}-1.45 \cdot \alpha_{\mathrm{xco}}^{2}\end{array}$ \\
\hline SER & $\begin{array}{l}59.98+7.11 \cdot \alpha_{\mathrm{cho}}^{2}-1.83 / \alpha_{\mathrm{xch}}^{2}+1.51 \cdot \cos ^{2}\left(\varphi_{\mathrm{xcho}}\right)- \\
-9.40 \cdot \mathrm{d}_{\mathrm{cx}}^{2}-63.76 / \mathrm{d}_{\mathrm{ho}}^{2}-1.95 \cdot \cos ^{2}\left(\varphi_{\mathrm{xcoh}}\right)-1.52 \cdot \alpha_{\mathrm{xco}}^{2}- \\
-3.44 / \alpha_{\mathrm{xco}}^{2}-2.25 \cdot \cos \left(\varphi_{\mathrm{xcho}}\right)-0.33 \cdot \mathrm{d}_{\mathrm{oc}}^{2}\end{array}$ \\
\hline THR & $\begin{array}{l}-17.61+46.26 / \mathrm{d}_{\mathrm{hx}}+0.28 \cdot \mathrm{d}_{\mathrm{oc}}^{2}-2.18 / \alpha_{\mathrm{xch}}^{2}+1.25 \cdot \cos ^{2}\left(\varphi_{\mathrm{xcoh}}\right)- \\
-0.23 / \alpha_{\mathrm{xco}}^{2}-1.58 \cdot \alpha_{\mathrm{xch}}^{2}+2.47 \cdot \cos \left(\varphi_{\mathrm{xcho}}\right)+27.37 / \mathrm{d}_{\mathrm{ho}}^{0.5}+ \\
+4.84 / \mathrm{d}_{\mathrm{cx}}^{0.5}-2.80 \cdot \cos ^{2}\left(\varphi_{\mathrm{xcho}}\right)\end{array}$ \\
\hline TRP & $\begin{array}{l}430.17-0.23 \cdot \mathrm{d}_{\mathrm{ch}}^{2}+0.89 \cdot \alpha_{\mathrm{xch}}^{2}+28.57 / \mathrm{d}_{\mathrm{ho}}^{2}- \\
-0.30 \cdot \cos ^{2}\left(\varphi_{\mathrm{xcho}}\right)+3.08 / \mathrm{d}_{\mathrm{cx}}^{2}-0.0006 / \cos ^{2}\left(\varphi_{\mathrm{xcho}}\right)+ \\
+0.11 \cdot \mathrm{d}_{\mathrm{oc}}^{2}+2.07 / \alpha_{\mathrm{xco}}^{2}+0.01 . / \cos \left(\varphi_{\mathrm{xcho}}\right)+34.69 / \mathrm{d}_{\mathrm{oc}}^{2}\end{array}$ \\
\hline VAL & $\begin{array}{l}951.75+29.47 / \mathrm{d}_{\mathrm{ch}}+1363.19 / \mathrm{d}_{\mathrm{ho}}^{2}+249.05 / \mathrm{d}_{\mathrm{oc}}^{2}+2.87 \cdot \alpha_{\mathrm{cho}}^{2}+ \\
+0.41 \cdot \mathrm{d}_{\mathrm{ch}}^{2}-10.32 \cdot \mathrm{d}_{\mathrm{ho}}^{2}+0.76 \cdot \mathrm{d}_{\mathrm{oc}}^{2}-1771.61 / \mathrm{d}_{\mathrm{ho}}^{0.5}\end{array}$ \\
\hline
\end{tabular}

